\title{
Deviations From Classical Hydrodynamic Theory in Highly Confined Planar Poiseuille Flow of a Polymer Solution
}

\author{
A.G. Menzel $^{1}$, P.J. Daivis ${ }^{1}$, B.D. Todd ${ }^{2}$ \\ ${ }^{1}$ School of Science and Centre for Molecular and Nanoscale Physics \\ RMIT University, GPO Box 2476, Melbourne, Victoria, 3001, Australia \\ E-mail: adrian.menzel@rmit.edu.au,peter.daivis@rmit.edu.au \\ ${ }^{2}$ Department of Mathematics, Faculty of Science, Engineering and Technology \\ Swinburne University of Technology, P.O. Box 218, Hawthorn, Victoria, 3112, Australia \\ E-mail: btodd@swin.edu.au
}

Received: 01 March 2017; revised: 28 March 2017; accepted: 28 March 2017; published online: 10 September 2017

\begin{abstract}
The behaviour of polymer solutions in highly confined geometries remains a subject of interest in rheology and fluid dynamics. In this paper, we investigate how well the classical hydrodynamic description based on the Navier-Stokes equations, Fourier's Law and Fick's Law describes the flow of a highly confined polymer solution. In particular, we examine the effects of depletion of polymer concentration at the wall-fluid interface and strain rate coupling to the heat flux. We present data from molecular dynamics simulations of a model polymer solution in explicit solvent undergoing planar Poiseuille flow for channel widths ranging from around 10 solvent atomic diameters to around 80 solvent atomic diameters. We find that the classical continuum approach works very well for channels wider than 20 solvent atomic diameters. For narrower channels, we observe deviations in the velocity, temperature and concentration profiles due to density oscillations near the walls, the polymer depletion effect, and possible weak strain rate coupling. For the narrowest channel, the wall effects extend to the centre of the channel but the underlying profiles are quite well described by the classical continuum picture. By allowing very long times of order $10^{4}$ reduced time units for relaxation to the steady state and averaging over very long runs of order $10^{5}$ reduced time units and 16 independent ensemble members, we are able to conclude that previously reported deviations from the classical continuum predictions (I.K. Snook, P.J. Daivis, T. Kairn, J. Physics-Condensed Matter 20, 404211 (2008)) were probably the result of insufficient equilibration time. Our results are also sufficiently accurate and precise to verify the expected quartic temperature profile predicted by classical hydrodynamic theory, with only a very small deviation which we can attribute to nonlinear coupling of the heat flux vector to the strain rate.
\end{abstract}

Key words: polymer, Poiseuille flow, velocity, temperature, channel, molecular dynamics

\section{INTRODUCTION}

Understanding the movement of polymer solutions through a channel is important to a range of processes like field flow fractionation, lubrication and injection moulding, as well as biological processes such as the flow of proteins and DNA in micro and nanofluidic devices [1].

Classically, confined fluids have been modelled using the Navier-Stokes equations and Fourier's law, which allows the prediction of velocity and temperature fields. The validity of this approach is widely recognised throughout all of fluid physics. However, previous molecular dynamics (MD) simulation results have suggested that the velocity and temperature profiles for highly confined polymer systems cannot adequately be described using the standard continuum approach [2]. Snook et al. [3] showed that polymer solutions 
experiencing planar Poiseuille flow in narrow channels exhibited velocity profiles that deviated from the prediction near the walls. For sufficiently narrow channels, these deviations may become highly significant and classical predictions of the velocity and temperature profiles will then be seriously inaccurate. Nanofluidic devices for the processing of macromolecular solutions, including protein and DNA solutions, can only be rationally designed if the flow can be modelled accurately, so these discrepancies need to be resolved.

Nonequilibrium molecular dynamics (NEMD) simulations using a sinusoidal transverse force (STF) by Baranyai et al. [4] and Ayton et al. [5] demonstrated evidence of heat flux in the absence of temperature gradients. MD simulations of planar Poiseuille flow by Todd and Evans [6] also showed that, even for a simple single component fluid, the temperature profile exhibited a significant quadratic deviation from the quartic profile predicted from Fourier's law. To explain this effect these studies postulated that the heat flux couples to the strain rate in the system, producing a quadratic deviation in the temperature profile.

Hess and Mansour [7] derived the strain rate coupling from the kinetic theory of gases and showed that it can explain deviations from classical temperature profiles in data obtained from direct simulation Monte Carlo. It is important to note, however, that the strain rate coupled contribution, in Hess' study, has the opposite sign to that seen in the work mentioned previously.

Travis and Gubbins [8] found no evidence of strain rate coupling in their molecular dynamics simulations of simple fluids. While they asserted that the existence of strain rate coupling is "unquestionable" they were sceptical of the magnitude of strain rate coupling found in the work by Todd and Evans [6].

Another current question in studies of polymer flow is whether or not the well known 'depletion effect' is dependent on the flow rate, or body force, applied to the system. Previous research by Kairn [2] could not demonstrate any dependence of the depletion on the body force. However, Ma and Graham [9] theoretically predicted this effect in planar Couette and Poiseuille flow of dilute polymer solutions.

In this paper, we compute the density, concentration, velocity and temperature profiles across the channel for a planar Poiseuille flow system simulated using in-house NEMD code. By fitting the classical continuum predictions to the velocity and temperature profiles we show that the classical treatment gives a good description for weak external fields and wide channels. From these fits, we extract effective viscosity and thermal conductivity coefficients. We then compare these fitted transport coefficients with linear transport coefficients obtained from homogeneous equilibrium simulations. The viscosity is also compared with the value obtained from homogeneous nonequilibrium simulations using the SLLOD algorithm.

This is the first time, to the authors knowledge, that data of such accuracy has been presented for polymer solutions, allowing us to comment on the subtle effects that exist in this system.

\section{METHOD AND THEORY}

For this study we used in-house molecular dynamics code that has been described previously [10]. Results for bulk polymer melt simulations performed by this program have previously shown good agreement with research in the literature $[11,12]$. This program explicitly solves the equations of motion for every particle in the system, including solvent particles, using Newtonian equations of motion.

All polymer beads and solvent atoms in this work interact with the Weeks-Chandler-Andersen (WCA) potential [13] which is a truncated and shifted version of the classic LennardJones (LJ) pair potential [14], given by

$$
\Phi_{\mathrm{WCA}}(r)= \begin{cases}\Phi_{\mathrm{LJ}}(r)+\epsilon & \text { if } r<2^{1 / 6} \sigma \\ 0 & \text { if } r \geq 2^{1 / 6} \sigma\end{cases}
$$

where,

$$
\Phi_{\mathrm{LJ}}(r)=4 \epsilon\left[\left(\frac{\sigma}{r}\right)^{12}-\left(\frac{\sigma}{r}\right)^{6}\right],
$$

$\epsilon$ is the potential energy well depth, $\sigma$ is the pairwise separation at which the LJ potential is zero, and $r$ is the pair separation distance. At the truncation point of the WCA potential, the force and the energy are both equal to zero. This means that the WCA potential is particularly convenient because it is short ranged and therefore computationally cheap and both the force and the energy go smoothly to zero at the truncation point.

All polymer beads and solvent atoms have the same mass $m$. Subsequently, all simulation parameters are given in terms of reduced units based on the characteristic length scale $\sigma$, energy scale $\epsilon$ and mass scale $m$ of this molecular model. The polymers are modelled as 20 -site freely jointed chains with each site being identical to a solvent atom. This model is simple enough to be computationally convenient and yet it includes the essential physics (excluded volume and noncrossability of the chains) for a realistic model of polymer dynamics $[10,11]$. The equations of motion for the various systems studied here are all solved using a 5th order GearPredictor-Corrector integration scheme [16].

In the subsections that follow, we discuss the methods used to obtain our results. Subsection A describes the simulations to determine the density, velocity, temperature and concentration profiles for the highly confined polymer solutions undergoing planar Poiseuille flow. Subsection B briefly summarises the well-established predictions of classical hydrodynamics used to fit the velocity and temperature profiles for planar Poiseuille flow. In subsection $\mathrm{C}$ we discuss the equilibrium simulation methods based on the Green-Kubo 
Tab. 1. Overview of system properties. $N_{\alpha}$ is the number of molecules of species $\alpha . \rho$ refers to the nominal input density of the simulation. For walled systems $T$ is the wall thermostat temperature, for homogeneous systems it is the fluid thermostat temperature. $c_{1}$ is the spatially averaged value of the polymer mass fraction. $L_{\beta}$ refers to the undeformed length of the total simulation box (including walls) where $\beta=x, y, z$. Shown in bold is the characteristic property for the associated system

\begin{tabular}{lllllllll}
\hline System ID & $N_{1}$ & $N_{2}$ & $\rho$ & $T$ & $c_{1}$ & $L_{x}$ & $L_{y}$ & $L_{z}$ \\
\hline GK & 80 & 6400 & 0.841 & 1.0163 & 0.2 & 22.82 & 22.82 & 18.26 \\
S0 & 0 & 8000 & 0.841 & 1.013 & $\mathbf{0}$ & 21.18 & 21.18 & 21.18 \\
S5 & 20 & 7600 & 0.841 & 1.013 & $\mathbf{0 . 0 5}$ & 21.18 & 21.18 & 21.18 \\
S10 & 40 & 7200 & 0.841 & 1.013 & $\mathbf{0 . 1}$ & 21.18 & 21.18 & 21.18 \\
S15 & 60 & 6800 & 0.841 & 1.013 & $\mathbf{0 . 1 5}$ & 21.18 & 21.18 & 21.18 \\
S21 & 82 & 6360 & 0.841 & 1.013 & $\mathbf{0 . 2 1}$ & 21.18 & 21.18 & 21.18 \\
S25 & 100 & 6000 & 0.841 & 1.013 & $\mathbf{0 . 2 5}$ & 21.18 & 21.18 & 21.18 \\
P83 & 84 & 6720 & 0.84 & 1 & 0.2 & 20.98 & $\mathbf{8 6 . 6 6}$ & 5.74 \\
P23 & 24 & 1920 & 0.84 & 1 & 0.2 & 20.98 & $\mathbf{2 7 . 3 4}$ & 5.74 \\
P11 $^{\dagger}$ & 12 & 960 & 0.84 & 1 & 0.2 & 20.98 & $\mathbf{1 5 . 4 7}$ & 5.74 \\
HP1 $^{\dagger}$ & $\mathbf{8 3}$ & 6720 & 0.84 & 1 & 0.1981 & 21.5 & 21.5 & 21.5 \\
HP2 $^{\dagger}$ & $\mathbf{8 4}$ & 6720 & 0.84 & 1 & 0.2 & 21.5 & 21.5 & 21.5 \\
HP3 $^{\dagger}$ & $\mathbf{8 5}$ & 6720 & 0.84 & 1 & 0.2019 & 21.5 & 21.5 & 21.5 \\
HS1 $^{\dagger}$ & 84 & $\mathbf{6 7 0 0}$ & 0.84 & 1 & 0.1995 & 21.5 & 21.5 & 21.5 \\
HS2 $^{\dagger}$ & 84 & $\mathbf{6 7 2 0}$ & 0.84 & 1 & 0.2 & 21.5 & 21.5 & 21.5 \\
HS3 $^{\dagger}$ & 84 & $\mathbf{6 7 4 0}$ & 0.84 & 1 & 0.2005 & 21.5 & 21.5 & 21.5 \\
\hline
\end{tabular}

${ }^{\dagger}$ Constant pressure simulation, with $P=7.39 . \rho$ and $L_{\beta}$ are average values.

formulae used to determine the viscosity, diffusion coefficient, thermal diffusion coefficient and thermal conductivity in multicomponent solutions. Because the Green-Kubo method is based on linear response theory it is guaranteed to yield the linear transport coefficients. However, it must be applied carefully to ensure that fully converged integrals are used to evaluate the transport coefficients. In this subsection we also discuss computation of the partial enthalpies, which are also required. Finally, in subsection D, we describe an alternative method to obtain the polymer solution viscosity from homogeneous nonequilibrium molecular dynamics simulations using the SLLOD algorithm. Evaluation of the linear (Newtonian) viscosity with this method requires extrapolation of the shear rate dependent viscosity to zero shear rate. The results of these simulations serve as an important consistency check on the Green-Kubo and planar Poiseuille flow values of the viscosity.

An overview of simulation parameters for all systems studied here can be seen in Tab. 1. In the system labels GK refers to the Green-Kubo system, $\mathrm{S}$ to the SLLOD systems, $\mathrm{P}$ to the Poiseuille flow systems, HP to the simulations to determine the polymer partial enthalpies, and HS to the simulations to determine the solvent partial enthalpies. The numbers refer to the polymer concentration percentage for the S systems and to the approximate fluid accessible channel width for the $\mathrm{P}$ systems. The numbers on HS and HP are simply sequential. Note that the index ' 1 ' for every property listed in Tab. 1 and throughout the rest of this work, refers to the polymer species, while ' 2 ' refers to the solvent species.

\section{1. Planar Poiseuille Flow}

We perform simulations on three different highly confined systems, subjected to an external body force in order to generate Poiseuille flow. The external body force produces Poiseuille flow without the need for a pressure gradient. The only difference between the two is that in the body force method, all of the fluid the properties, including the pressure, are conveniently uniform in the flow direction because the fluid is effectively in free fall. All three systems consist of a polymer solution ( $20 \%$ by mass concentration) confined in the $y$ direction by two atomic walls. Each system is created with a different separation between the two walls, allowing us to investigate the effects of different levels of confinement.

In each system both walls consist of 228 particles each arranged in 2 hexagonal-close-packed layers. We have found that two layers for each wall is sufficient to provide adequate temperature control and prevent fluid penetrations of the walls. The nominal input mass density of the fluid is $\rho=0.84$. Because the reduced mass of each polymer site and solvent atom in the system is 1 , the mass density is equal to the number density. Periodic boundary conditions (PBCs) are used to extend the simulation box in the $x$ (flow) direction and $z$ directions to provide an effectively infinite planar channel for the solution to flow in. For a visualisation of this system, see Fig. 1.

The equations of motion for the fluid are given by:

$$
\dot{\mathbf{r}}_{i \alpha}=\frac{\mathbf{p}_{i \alpha}}{m}
$$


and

$$
\dot{\mathbf{p}}_{i \alpha}=\mathbf{F}_{i \alpha}^{W C A}+\mathbf{F}_{i \alpha}^{C}+m_{i \alpha} \mathbf{F}^{e} .
$$

In these equations the subscript $\alpha$ refers to the $\alpha$ th particle on the $i$ th molecule, $\dot{\mathbf{r}}$ is the velocity and $\mathbf{p}$ is the momentum, both in the laboratory frame and $m$ is the polymer site or solvent atom mass. The rate of change in momentum, $\dot{\mathbf{p}}$, is given by an appropriate sum of forces on the particle. $\mathbf{F}^{C}$ is the sum of constraint-type forces on the particle and, as such, is zero for solvent atoms. $m \mathbf{F}^{e}$ is the gravity-like external body force applied to all particles in the system to induce Poiseuille flow, and $\mathbf{F}^{W C A}$ is the sum of WCA pair-interaction forces on the particle (see Eqn. 1).

Polymer bond lengths are rigidly constrained by applying a force in Eqn. $4, \mathbf{F}^{C}$, that satisfies the constraint condition, $\left|\mathbf{r}_{i j}\right|=\left|\mathbf{r}_{j}-\mathbf{r}_{i}\right|=\sigma=1$.

We note that, given our potential and equations of motion, the polymer sites are identical to solvent sites, with the exception that the polymer sites experience intra-molecular constraint forces while the solvent sites do not.

The equations of motion for the walls are modified to include a Gaussian thermostat and a constraint on the centre of mass of the wall layers to prevent them from moving;

$$
\dot{\mathbf{r}}_{i}=\frac{\mathbf{p}_{i}}{m}
$$

and

$$
\dot{\mathbf{p}}_{i}=\mathbf{F}_{i}^{W C A}+\mathbf{F}_{i}^{W}-\alpha_{\beta} \mathbf{p}_{i}+\lambda_{\beta} \hat{\mathbf{y}},
$$

where $\mathbf{F}_{i}^{W}=-k\left(\mathbf{r}_{i}-\mathbf{r}_{i}^{e q}\right)$ is a harmonic force used to tether the wall particles to their equilibrium positions, $\mathbf{r}_{i}^{e q}$, with $k=57.15$. This value of $k$ has previously been shown to provide good thermal coupling to the fluid $[2,3] . \alpha_{\beta}$ is a Gaussian isokinetic thermostat applied to each wall layer, and $\lambda_{\beta}$ is a constraint applied to each wall layer to keep the $y$ position of the $\beta$ th layer's centre-of-mass constrained to its initial value.
Finally, the equations of motion are solved using a 5th order Gear predictor-corrector integrator with a reduced timestep, $\delta t$, of 0.001 .

We drive the polymer solution to flow by introducing a gravity-like external force field in the $x$-direction, $F_{x}^{e}$, into the equation of motion for the momentum given by Eqn. 4 . For this study we have used different values of $F_{x}^{e}$ for each system in order to achieve approximately the same magnitude of velocity at the channel centre.

We equilibrated these systems for over $10^{7}$ time steps ( $10^{4}$ reduced time units) each. Once these systems have reached a steady state we compute histograms of the density, concentration, velocity and temperature profiles across the channel and ensemble average over 16 independent systems each sampled every 20 steps for an accumulation period greater than $10^{8}$ time steps $\left(10^{5}\right.$ reduced time units) in each case. These profiles were computed using 400 bins across the channel, giving bin resolutions ranging from approximately 0.2 atomic diameters to approximately 0.04 atomic diameters depending on the channel width.

To retrieve the temperature profile we compute the molecular centre of mass total kinetic energy density, $K=(2 V)^{-1} \sum_{i}^{N_{m o l}} \mathbf{p}_{i} \cdot \mathbf{p}_{i} / m_{i}$, the momentum density in the direction of flow, $J_{x}$, which is the $x$-component of the molecular centre of mass momentum density vector given by $\mathbf{J}=V^{-1} \sum_{i}^{N_{m o l}} m_{i} \mathbf{v}_{i}$, the molecular number of degrees of freedom density, $f=3 V^{-1} N_{m o l}$, and the mass density, $\rho=V^{-1} \sum_{i}^{N_{m o l}} m_{i}$. Note that $m_{i}$ is the mass of the $i$ th molecule, $m_{i}=\sum_{\alpha} m_{i \alpha}$, which for atomic species is the same as the mass of the atom. We then post-process the data to compute the temperature from the thermal kinetic energy, using the following equation on a per-bin basis:

$$
k_{B} T_{k}=\frac{2 K_{k}-J_{x, k}^{2} / \rho_{k}}{f_{k}}
$$

where the subscript $k$ is the corresponding bin across the channel. We use this method instead of calculating the temperature

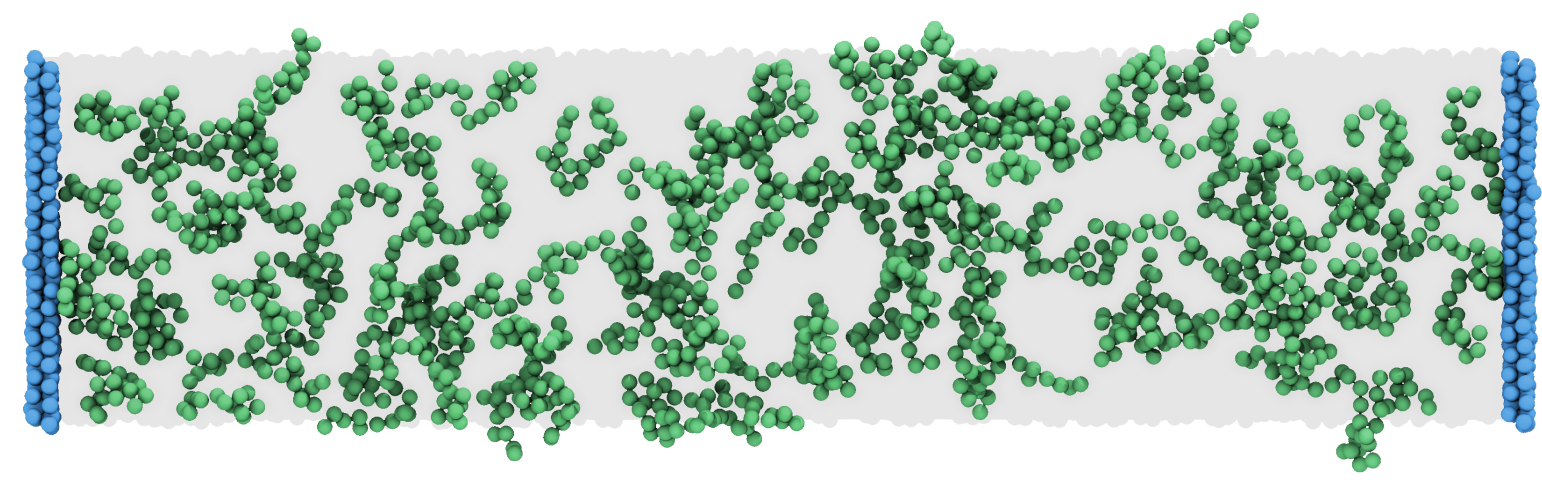

Fig. 1. Snapshot of simulated system, $L_{y} \approx 86, W \approx 83$. Solvent atoms are shown as a grey continuum, polymer atoms are shown between the walls in green, while the walls to either side are shown in blue. In this image, the $x$-direction is vertical, the $y$-direction is oriented horizontally, and the $z$-direction on the axis that is into the page 
instantaneously using a fitted equation for the streaming velocity. This ensures that we make no assumptions about the functional form of the velocity profile, and that we can approach the ensemble average limit by simply increasing the accumulation time.

We present the results for molecular centre-of-mass velocities and temperatures in this work. While atomic versions of this temperature calculation can be constructed, the atomic version of the momentum density does not adequately account for the streaming angular momentum of the sites in the system. To compensate for this one must be able to calculate the correct streaming angular momentum densities in a bin and subtract those components from the total kinetic energy density. The correct determination of both the number and localisation of the atomic degrees of freedom in a constrained molecule is also non-trivial. Calculating temperatures in the molecular representation circumvents these concerns.

\section{2. Continuum Predictions for Planar Poiseuille Flow}

From continuum mechanics we can obtain equations for the velocity and temperature by integrating appropriately simplified balance equations.

Assuming constant viscosity and density throughout the fluid, the continuum prediction for the velocity profile across the channel in the steady state is given by:

$$
v_{x}(y)=-\frac{\rho F_{x}^{e}}{2 \eta_{0}}\left(y^{2}-\frac{W^{2}}{4}\right)+v_{w}
$$

where $\rho$ is the nominal mass density of the fluid, $F_{x}^{e}$ is the external field in the $x$-direction, $\eta_{0}$ is the zero strain viscosity, $y$ is the position in the channel in the $y$-direction, with the centre of the channel located at $y=0, W$ is the width of the channel accessible to the fluid and $v_{w}$ is the velocity at the walls.

Neglecting the effects of thermal diffusion (which are often assumed to be small or negligible) and assuming that the thermal conductivity is constant the continuum prediction for the temperature profile in the steady state is given by,

$$
T(y)=-\frac{\rho^{2}\left(F_{x}^{e}\right)^{2}}{12 \eta \lambda}\left(y^{4}-\frac{W^{4}}{16}\right)+T_{w},
$$

where $\lambda$ is the fluid thermal conductivity and $T_{w}$ is the temperature at the walls.

These equations assume that the centre of the channel is at $y=0$. They do not account for shear rate, concentration or temperature dependence of the transport coefficients or the density. Therefore these effects must be small for them to be valid.

For systems P83, P23, and P11 the accessible channel widths are $W=82.5 \pm 0.1,23.15 \pm 0.04$ and $11.33 \pm 0.02$ respectively, determined by the distance between the first and last bins to have significant non-zero values of the density (greater than $10 \%$ of the bulk value).
To calculate the velocity and temperature profiles we must obtain values for the viscosity and thermal conductivity. These can be calculated using Green-Kubo relations. The viscosity can also be computed by direct calculation and extrapolation to zero strain rate in homogeneous NEMD simulations. In principle, the thermal conductivity can also be computed by NEMD methods using the Evans-Cummings heat flow algorithm and the colour diffusion algorithm [15], but they have not been implemented in this work.

\section{3. Transport Coefficients From Equilibrium}

To calculate the zero strain rate viscosity the following Green-Kubo [17, 18] relation can be used,

$$
\eta_{0}=\int_{0}^{\infty} G(\tau) d \tau
$$

where [19],

$$
G(\tau)=\frac{V}{10 k_{B} T}\left\langle\mathbf{P}^{\mathrm{ts}}(0): \mathbf{P}^{\mathrm{ts}}(\tau)\right\rangle,
$$

$V$ is the volume of the simulation box, $k_{B}$ is Boltzmann's constant, $T$ is the temperature of the fluid, and $\mathbf{P}^{\mathrm{ts}}$ is the traceless symmetric part of the atomic pressure tensor.

For multicomponent systems the Green-Kubo relations do not permit calculation of the thermal conductivity directly. Instead we must compute the following phenomenological coefficients,

$$
\begin{aligned}
& L_{q q}=\frac{V}{3 k_{B}} \int_{0}^{\infty}\left\langle\mathbf{J}_{q}(0) \cdot \mathbf{J}_{q}(\tau)\right\rangle d \tau, \\
& L_{11}=\frac{V}{3 k_{B}} \int_{0}^{\infty}\left\langle\mathbf{J}_{1}(0) \cdot \mathbf{J}_{1}(\tau)\right\rangle d \tau,
\end{aligned}
$$

and

$$
L_{1 q}=\frac{V}{3 k_{B}} \int_{0}^{\infty}\left\langle\mathbf{J}_{1}(0) \cdot \mathbf{J}_{q}(\tau)\right\rangle d \tau,
$$

where $\mathbf{J}_{1}$ is the instantaneous mass flux vector of species 1 and $\mathbf{J}_{q}$ is the instantaneous unprimed heat flux vector.

These phenomenological coefficients can be related to transport coefficients through the following relations [20];

$$
D^{\prime}=\frac{L_{1 q}}{\rho c_{1} c_{2} T^{2}},
$$

which is the thermal diffusion coefficient and,

$$
D_{m}=\frac{L_{11}}{\rho c_{2} T}\left[\frac{\partial \mu_{1}}{\partial c_{1}}\right]_{T, P},
$$

which is the mutual diffusion coefficient. Here, $\mu_{\alpha}$ is the chemical potential of species $\alpha, c_{\alpha}$ is the mass fraction of species $\alpha$, subsequently referred to as 'concentration'. 
For the thermal conductivity we must used the primed phenomenological coefficient [20], $L_{q q}^{\prime}$, given by;

$$
L_{q q}^{\prime}=L_{q q}-2 L_{1 q}\left(h_{1}-h_{2}\right)+L_{11}\left(h_{1}-h_{2}\right)^{2},
$$

where $h_{\alpha}$ is the partial enthalpy of species $\alpha$. This primed coefficient takes into account the transport of heat due to thermal diffusive fluxes and mass fluxes and is related to the corresponding transport coefficient, the thermal conductivity, by,

$$
\lambda=\frac{L_{q q}^{\prime}}{T^{2}} .
$$

\section{4. Viscosity from Homogeneous Shear}

We can also calculate the viscosity from simulations of homogeneous systems experiencing constant strain rate. These simulations can be performed using the SLLOD equations of motion [21,22], which permit the simulation of constant strain rate in a periodically infinite fluid and are given by,

$$
\dot{\mathbf{r}}_{i \alpha}=\mathbf{c}_{i \alpha}+\mathbf{r}_{i} \cdot \nabla \mathbf{v},
$$

and

$$
m_{i \alpha} \dot{\mathbf{c}}_{i \alpha}=\mathbf{F}_{i \alpha}-m_{i} \mathbf{c}_{i} \cdot \nabla \mathbf{v}-\alpha_{K} m_{i} \mathbf{c}_{i},
$$

where $\mathbf{F}_{i \alpha}$ is the sum of all interatomic and constraint forces acting on site $\alpha$ of molecule $i$ and $\alpha_{K}$ is the thermostat multiplier for the Gaussian constraint thermostat that keeps the kinetic temperature of the molecular centres of mass fixed. Here we have used $\mathbf{c}$ to denote the thermal velocity also called the peculiar velocity, where the streaming component of the velocity is explicitly stated in the first equation as $\mathbf{r}_{i} \cdot \nabla \mathbf{v}$. This is in contrast to the previously stated equations of motion where the streaming velocity is not explicitly known and $\mathbf{p}$ refers to the total, 'laboratory', momentum. Also note that the shearing deformation and thermostat are applied to the molecular centres of mass. These equations allow the calculation of various properties evaluated at a particular strain rate, which can then be extrapolated back to a zero strain rate value.

To calculate the strain rate dependent viscosity we use the following equation:

$$
\eta(\dot{\gamma})=-\frac{P_{y x}}{\dot{\gamma}}
$$

We then extrapolate this value back to zero strain rate to determine the zero strain rate viscosity for a particular state point,

$$
\eta_{0}=\lim _{\dot{\gamma} \rightarrow 0} \eta(\dot{\gamma})
$$

\section{RESULTS}

\section{1. Transport Coefficients}

Using the Green-Kubo relations given by Eqns. 10-14 we were able to calculate the phenomenological and transport coefficients for this system.

The Green-Kubo system consisted of 8320 -site polymer chains in solution with 6400 WCA atoms, each with mass 1 , for a total site number and mass concentration of 0.2 . The state point of this system was created to match the centre channel state point obtained from preliminary results for the strongest body force applied to the widest channel, system P83 (see Tab. 1).

The partial enthalpies required to calculate $L_{q q}^{\prime}$ using Eqn. 17 are determined by creating a similar system and then varying the number of molecules of one species while keeping the number of molecules of the other species, and the pressure, constant. We then calculate the enthalpy at that state point and use these values to find a finite difference approximation to the partial enthalpies at the relevant state point. These results can be found in Tab. 2 .

Using Eqn. 17 and Eqn. 18 with $\mathrm{T}=1.0163$, we find that $\lambda=6.26 \pm 0.16$.

Fig. 2 shows the integral of the stress autocorrelation function. It converges to a value of $\eta_{0}=3.68 \pm 0.03$, after approximately 275 reduced time units. The stress is a very slowly relaxing property for non-dilute polymeric fluids, even for chain lengths of only 20 monomers.

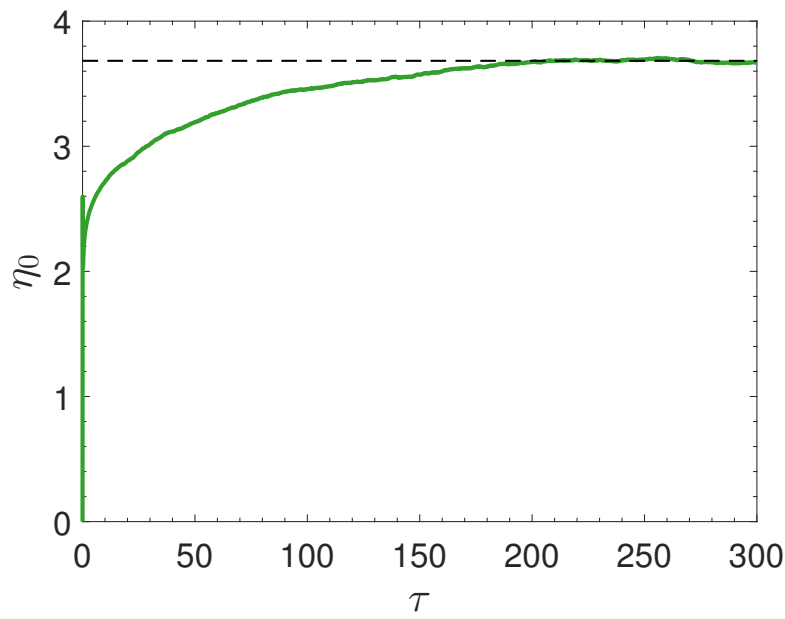

Fig. 2. Plot of the normalized cumulative integral of the stress autocorrelation function for system GK (see Tab. 1) against the delay time of the correlation function in reduced time units. The dashed line gives the extracted value of viscosity, $\eta$

We also created a variety of systems at a similar state point but different concentrations and induced the system to flow using the SLLOD equations of motion, given by Eqn. 19 and Eqn. 20. We then calculated the strain rate dependent viscosity for each concentration, and extrapolated the value to the zero strain rate limit. Presented in Fig. 3 is the zero 
strain rate viscosity as a function of concentration for the range explored by this work.

Tab. 2. Properties calculated from homogeneous simulations

\begin{tabular}{lll}
\hline System & Property & Value \\
\hline GK & $L_{22}\left(=L_{11}\right)$ & $1.79 \pm 0.03 \times 10^{-2}$ \\
GK & $L_{2 q}\left(=-L_{1 q}\right)$ & $2.72 \pm 1.09 \times 10^{-2}$ \\
GK & $L_{q q}$ & $6.51 \pm 0.10$ \\
GK & $L_{q q}^{\prime}$ & $6.47 \pm 0.16$ \\
GK & $\lambda$ & $6.26 \pm 0.15$ \\
HP1-3 & $h_{1}$ & $10.0 \pm 0.1$ \\
HS1-3 & $h_{2}$ & $11.4 \pm 0.05$ \\
GK & $\eta_{0}$ & $3.69 \pm 0.03$ \\
S0-25 & $\eta_{0}$ & $3.66 \pm 0.04$ \\
\hline
\end{tabular}

The equation:

$$
\eta_{0}=2.15+6.19 c_{1}+7.03 c_{1}^{2}
$$

gives a second order polynomial equation of best fit, in a least squares sense, to this data.

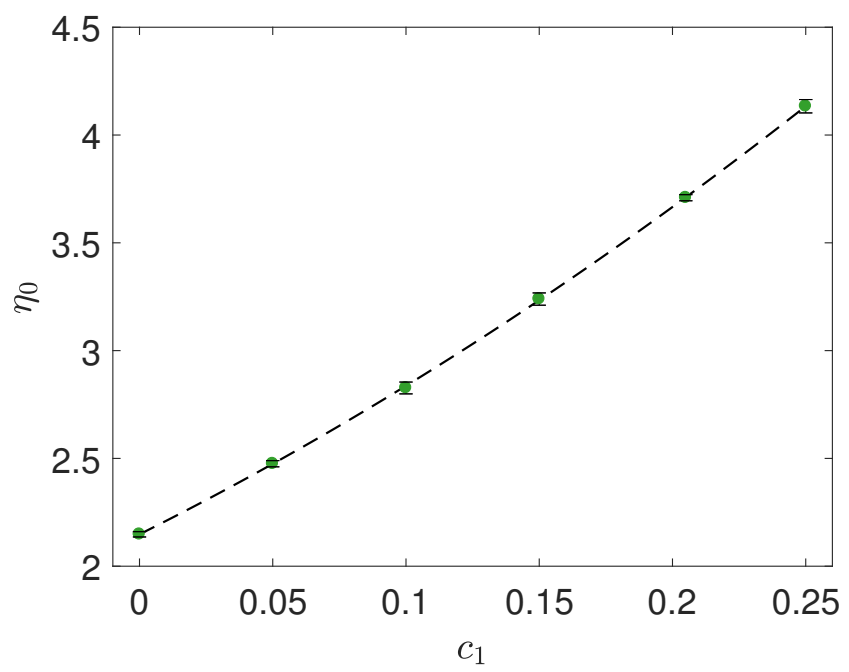

Fig. 3. Plot of the zero strain rate extrapolated viscosity, as a function of concentration. The dashed line gives a 2 nd order polynomial least squares fit to the data, shown by Eqn. 23

For details of these systems see IDs S0 to S25 in Tab. 1.

\section{2. Concentration Profiles}

Before presenting the results for the velocity and temperature profiles it is important to discuss the behaviour of the density and polymer concentration across the channel, as these factors have a strong effect on the local transport properties of the fluid.
Fig. 4 shows the concentration profile across the channel for systems P83 (left), P23 (middle), and P11 (right). This concentration is a mass fraction defined as, for species $\alpha$,

$$
c_{\alpha}=\frac{\rho_{\alpha}}{\rho_{1}+\rho_{2}} .
$$

All confined systems shown here demonstrate a strong depletion effect near the walls. One way to explain this depletion is that it is caused by the large entropy cost for a polymer to exist near the wall due to the lower entropy of the permitted conformations in that region [23].

In system P83 (left), for the three lowest body force values, $F_{x}^{e}=0,0.0001$, and 0.0005 the behaviour of the concentration essentially does not change. However as the body force increases to 0.001 we see a shear-mediated increase of the polymer concentration in the centre of the channel, which corresponds to an increase in the magnitude of the depletion effect near the wall.

Similarly, in the P23 system (middle) the concentration profile is mostly unaffected for the two lowest body force values, $F_{x}^{e}=0$, and 0.001 . However as the body force increases to $F_{x}^{e}=0.005$ and 0.01 we see an increasingly depleted near-wall region and an increasingly enhanced concentration in the centre region.

The pattern is the same in the P11 system (right), with the three lowest body forces seemingly having little effect on the concentration before the highest, $F_{x}^{e}=0.03$, starts to deplete the polymer more thoroughly near the walls.

In the absence of an external field, the perturbation to the polymer concentration due to the presence of the walls is a function only of the wall-fluid potential. The wall and fluid are identical in each of the three systems, therefore the perturbation due to the presence of the wall is also identical. As the width of the channel decreases, the displaced polymer molecules have less unperturbed channel space to retreat towards. This leads to an increasing polymer concentration in the channel centre, resulting in an increased local viscosity as given by Eqn. 23 .

For the P83 system the channel centre concentration is only slightly above the nominal polymer concentration, $c_{1}=0.20$, at $c_{1} \approx 0.21$, while for the P23 and the P11 systems the channel centre concentration rises to $c_{1} \approx 0.22$ and $c_{1} \approx 0.24$ respectively. This corresponds to a significant increase in the channel centre viscosity.

Fig. 3 shows that the corresponding viscosity values for concentrations of $c_{1}=0.2,0.21,0.22$, and 0.24 , are $\eta_{0}=3.67,3.75,3.85$, and 4.03 respectively. It is important to account the increase in effective concentration when calculating predictions for the velocity and temperature profiles.

Another interesting feature of these concentration profiles is the periodic bumps in the profile. These bumps are due to density oscillations and atomic packing effects in the fluid. 

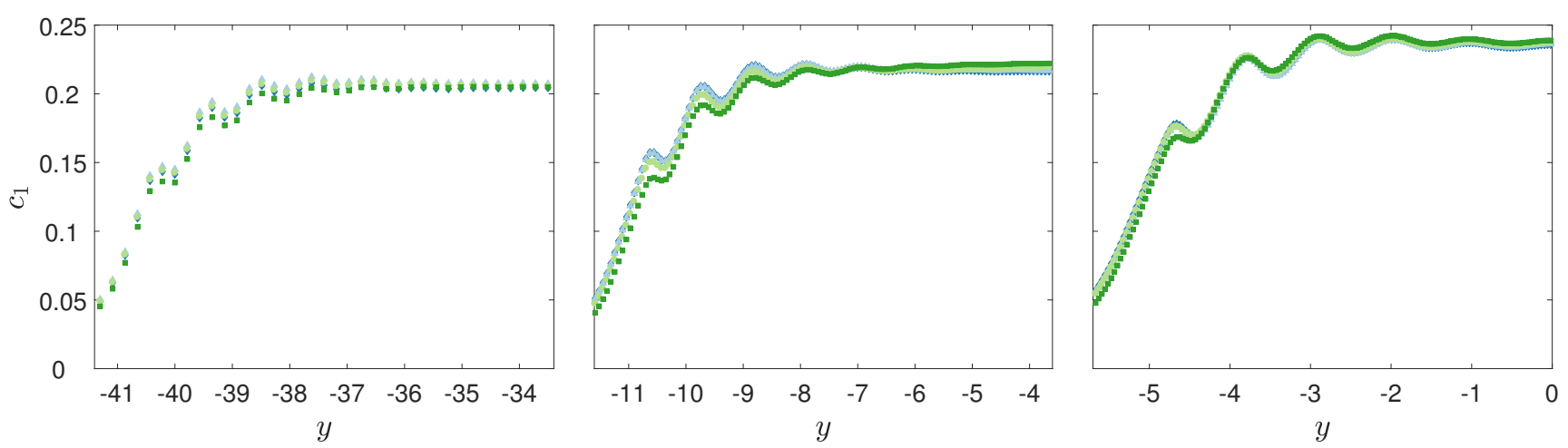

Fig. 4. Plot of the polymer mass concentration profile near the left wall for systems P83 (left), P23 (middle) and P11 (right) for various body force values. The values for lowest to highest body force are given by dark blue diamonds, light blue triangles, light green circles, and dark green squares. For most of the plots, all except the highest body force (dark green squares) lay on top of each other. For P83, $F_{x}^{e}=0$, $0.0001,0.0005,0.001$, for P23, $F_{x}^{e}=0,0.001,0.005,0.01$, and for P11, $F_{x}^{e}=0,0.003,0.015,0.03$

\section{3. Velocity Profiles}

Before presenting results for the temperature profiles we must verify that the velocity profiles are well described by the zero strain rate viscosities obtained previously.

Fig. 5 shows the molecular centre-of-mass velocity profile across the channel for systems P83 (left), P23 (middle) and P11 (right). We fit polynomial equations of increasing order to the velocity profiles, stopping when we retrieve random residuals. The highest order fit to the velocity profiles is given by:

$$
v_{x}(y)=k_{0}+k_{2} y^{2}+k_{4} y^{4}+k_{6} y^{6}+k_{8} y^{8}
$$

where $k_{0}$ is the shift in the velocity profile and $k_{2}=$ $-\rho F_{x}^{e} / 2 \eta_{0}$. From this, we are able to extract a value for the viscosity via comparison with Eqn. 8. Here, we assume that the coefficient of the quadratic component is directly related to the zero strain rate viscosity. This requires that the effect of the second order term is only a result of the linear transport equations. This also assumes that the fitted equation is sufficient to describe the velocity profile, which is evidenced by random residuals.

In Fig. 5, for system P83 (left), the fitted values for the viscosity for $F_{x}^{e}=0.001,0.0005$, and 0.0001 are $\eta_{0}=3.66 \pm 0.1,3.66 \pm 0.1$, and $3.78 \pm 0.1$ respectively. These values show very good agreement with the Green-Kubo value, $\eta_{0}=3.69 \pm 0.03$, and SLLOD value, $3.66 \pm 0.04$, for the viscosity. It is important to note that the parameter for the velocity at the wall in Eqn. 8 is a free parameter for this fit.

The uncertainties given for both the fitted viscosity, and fitted thermal conductivity, presented in this work have been obtained by varying the order of the equation fitted to the data. Uncertainties obtained only from the mean squared error and degrees of freedom error in the fitting parameters only account for the random error and are vastly underestimated.

Closer examination of the data shown in Fig. 5 for system P83 shows that near the wall there is a slight difference between the gradients of the fitted equation and the data. This system lacks any strong effects due to density oscillations that could significantly disturb the velocity near the walls. There-
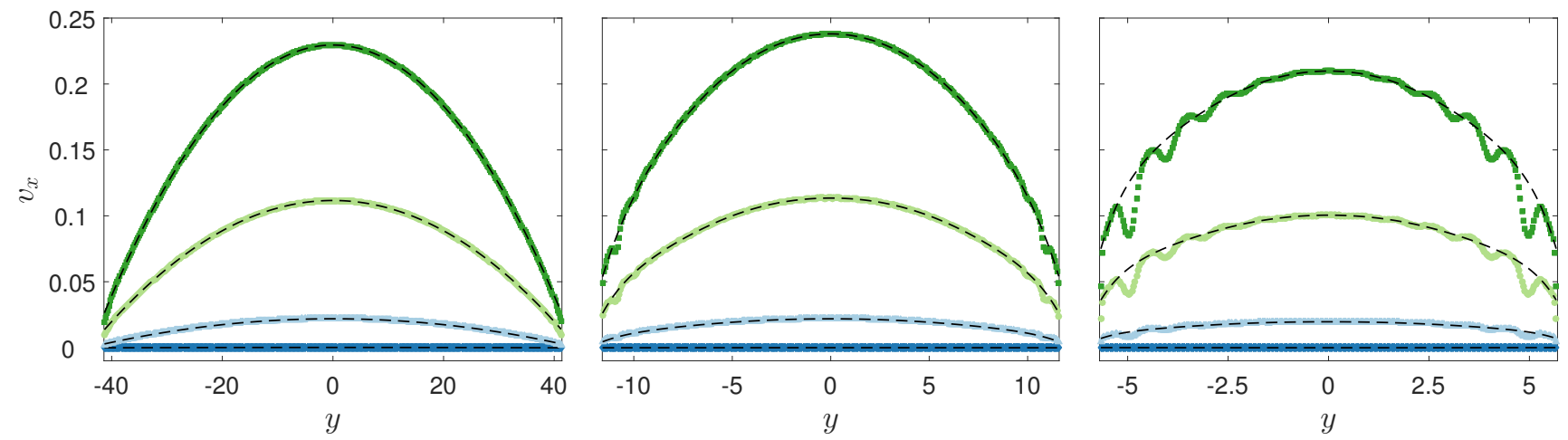

Fig. 5. Plot of the molecular centre-of-mass velocity profiles of the fluid in systems P83 (left), P23 (middle), and P11 (right), for the 4 different body forces applied to those systems. For P83, $F_{x}^{e}=0,0.0001,0.0005,0.001$, for P23, $F_{x}^{e}=0,0.001,0.005,0.01$, and for P11, $F_{x}^{e}=0,0.003,0.015,0.03$. The increasing forces correspond to larger magnitudes of effect in the plot. The dashed line gives an even polynomial fit of various orders up to the 8th order (Eqn. 25) for the largest applied body force 

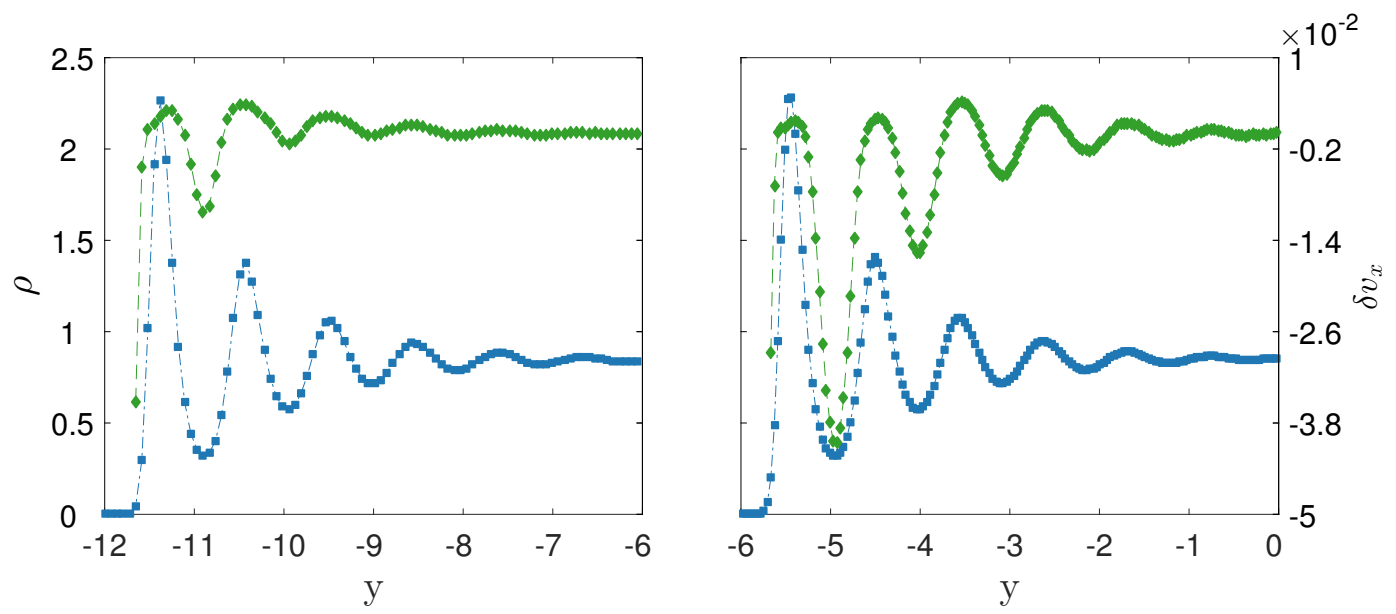

Fig. 6. Plot of the density profile (squares, left axis) and residuals of fitted velocity profile (diamonds, right axis) for system P23 \& $F_{x}^{e}=0.01$ (left) and system $\mathrm{P} 11 \& F_{x}^{e}=0.03$ (right). Lines are included only to guide the eyes

fore this deviation is likely due to the significant depletion of concentration near the wall, as seen in Fig. 4, which would significantly change the local value of the viscosity given by Eqn. 23.

For the largest body force, $F_{x}^{e}=0.001$, near the wall the concentration of the polymer is, at most, $c_{1}=0.05$ corresponding to a zero strain rate viscosity of $\eta_{0}=2.47$ as compared to the fitted viscosity $\eta_{0}=3.66$. The P23 and P11 systems near-wall regions are dominated by the effects of the density oscillations and so this feature is difficult to observe in those systems.

The gradient of the velocity profile (i.e. the strain rate), is given by,

$$
\dot{\gamma}=-\frac{\rho F_{x}^{e}}{\eta_{0}} y .
$$

Which, for the fitted viscosity gives $\dot{\gamma}(y=-41.3)=$ 0.0095. If, instead, we used the wall-adjacent concentration, $c_{1}=0.05$, to determine the viscosity using Eqn. 23, as an estimate to the local viscosity in this region we obtain $\dot{\gamma}(y=-41.3)=0.0140$. This compares well with the gradient obtained from the data of $\dot{\gamma}(y=-41.3)=0.0145$, which was determined by a backwards difference estimate of the velocity gradient using the first two data points at $y=-41.3$ and -41.08 .

For system P23 (middle) the corresponding values of the fitted values of the viscosity for $F_{x}^{e}=0.01,0.005$, and 0.001 are $\eta_{0}=3.88 \pm 0.4,4.21 \pm 0.4$, and $4.14 \pm 0.4$. These values show good agreement with the zero strain rate viscosity obtained from SLLOD with $\eta_{0}\left(c_{1}=0.22\right)=3.85$ from Eqn. 23.

The velocity near the wall in this system is strongly affected by the density oscillations. Fig. 6 shows the residuals of the fit to the velocity profile near the left wall overlaid with the density oscillations. The residuals show strong deviations from a purely polynomial description which, with the exception of the first peak, line up well with the density profile oscillations. The presence of polymer depletion and velocity slip, in addition to the density oscillations are the most likely reasons the first residual peak is not aligned with the first density peak.

Likewise, for System P11 the fitted equation seems to fit the overall trend of the data well, and the corresponding values of the fitted viscosity for $F_{x}^{e}=0.03,0.015$, and 0.003 are $\eta_{0}=4.2 \pm 1,4.42 \pm 1$, and $4.8 \pm 1$. These values, although uncertain, compare relatively well with the value of zero strain rate viscosity obtained from SLLOD, $\eta\left(c_{1}=0.24\right)=4.04$, given by Eqn. 23.

For the narrowest channel, strong density oscillations are present across the entire channel width. We see the characteristic velocity oscillations almost across the entire channel.

The perturbative force, the local density profile, is almost identical between the P23 and P11 systems, and yet the magnitude of the perturbation on the velocity is much higher in the P11 system. These systems have the same walls, same fluid and approximately same magnitude of the velocity profile and oscillations in the density profile. Whatever coupling exists between the velocity and density it must, therefore, be proportional to either the applied external field, or possibly the gradient of the strain rate in the system, as these are the only significant characteristic differences between the different systems. In such a case, a larger external force, or steeper strain rate gradient, corresponds to a stronger coupling to the density oscillations.

Overall we find that the continuum prediction for the velocity profile is accurate to first order. In the P83 system, the fit could be further improved by changing from the constant viscosity to a local viscosity across the channel, which would account for the deviation in the gradient near the walls. For 

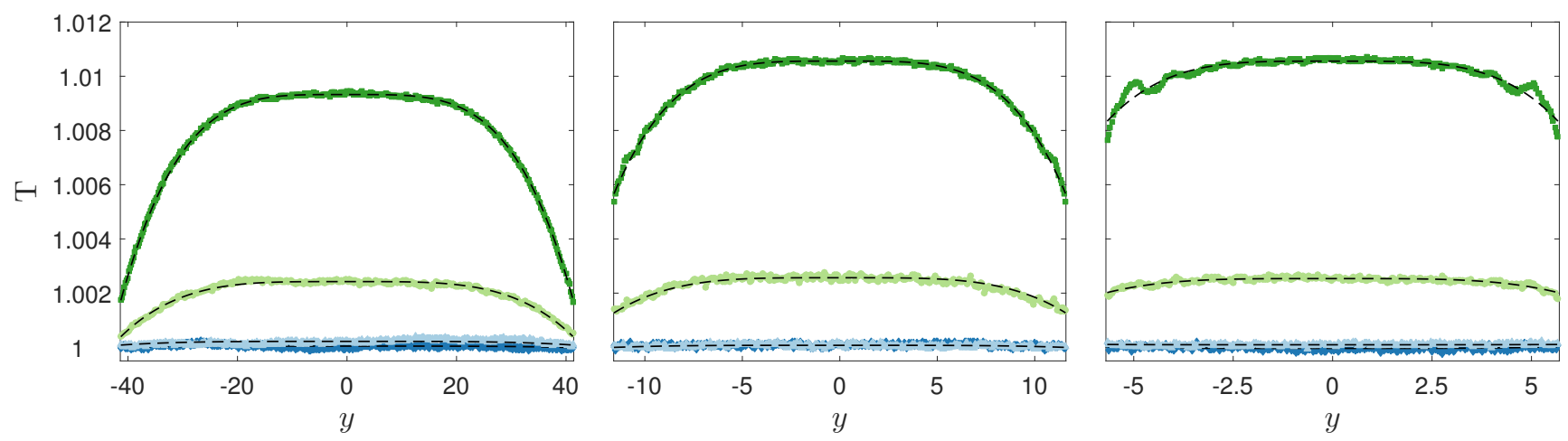

Fig. 7. Plot of the molecular centre-of-mass temperature profiles of the fluid in systems P83 (left), P23 (middle), and P11 (right), for the 4 different body forces applied to those systems. For P83, $F_{x}^{e}=0,0.001,0.0005,0.0001$, for P23, $F_{x}^{e}=0,0.01,0.005,0.001$, and for P11, $F_{x}^{e}=0,0.03,0.015,0.003$. The increasing forces correspond to larger magnitudes of effect in the plot. The dashed line gives a quartic fit to the profiles

the P23 and P11 systems the near-wall region is dominated by density oscillations, obscuring the effect of polymer depletion on the local viscosity. In these systems, the continuum prediction could be improved by finding an appropriate nonlocal coupling of the velocity profile to the density, which should include either the magnitude of the applied external field or possibly the gradient of the strain rate. The theoretical framework for this has already been discussed by Glavatskiy, Dalton et al. [24, 25].

\section{4. Temperature Profiles}

Having predicted an acceptable value for the zero strain rate viscosity, $\eta_{0}$, for each channel width we can now fit equations to the temperature profiles to retrieve the thermal conductivity, $\lambda$.

Fig. 7 shows the molecular centre of mass kinetic temperature across the channel for each value of the body force for system P83 (left), P23 (middle) and P11 (right). By fitting
Eqn. 9 to the data we were able to extract a value for the thermal conductivity. For the viscosity parameter, we used the values obtained from SLLOD simulations given by Eqn. 23, with $c_{1}=0.21,0.22$, and 0.24 for P83, P23, and P11 respectively. In each system, the magnitude of the effect due to the smallest body force is so small as to be barely indistinguishable from the equilibrium system, which has no temperature gradient. Therefore we only present values for the thermal conductivity from the data obtained from the two largest body forces applied to each system.

The values of the thermal conductivity from the fitted equation are; For P83 and body forces $F_{x}^{e}=0.001$, and $0.0005, \lambda=6.0 \pm 0.1$ and $5.6 \pm 0.1$. For P23 and body forces $F_{x}^{e}=0.01$, and $0.005, \lambda=5.6 \pm 0.7$, and $5.3 \pm 0.7$. For P11 and body forces $F_{x}^{e}=0.03$, and $0.015, \lambda=6 \pm 2$ and $\lambda=6 \pm 2$.

These values agree relatively well with the value calculated from equilibrium Green-Kubo, $\lambda=6.5 \pm 0.1$ with
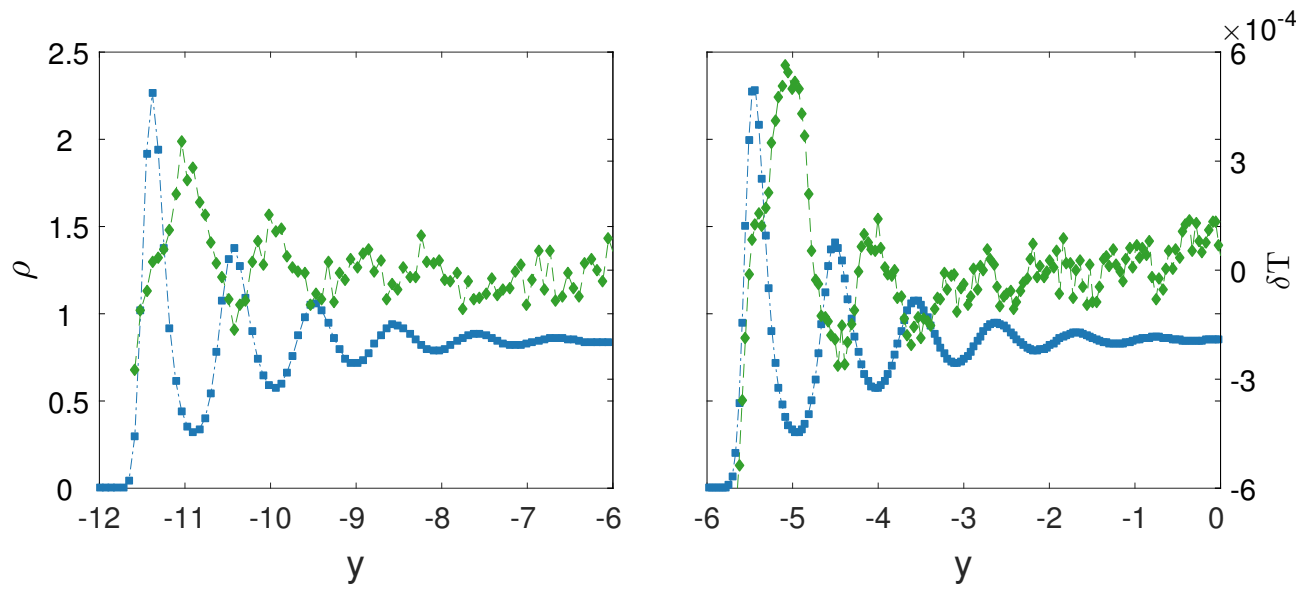

Fig. 8. Plot of the density profile (squares, left axis) and residuals of fitted temperature profile (diamonds, right axis) for system P23 \& $F_{x}^{e}=0.01$ (left) and system P11\& $F_{x}^{e}=0.03$ (right). Lines are included only to guide the eyes 
$c_{1}=0.2$. Since the temperature profile is so well fitted by Eqn. 9 estimating the uncertainties via change of the fitting equation likely leads to underestimated uncertainties. The standard error in the fitting parameters due to to the mean squared error and degrees of freedom error in the fit itself is also extremely small and likely to underestimate the uncertainties. However, this assumes that all of the data points in the temperature profile are independent. This is clearly not the case, because the noise in neighbouring data points is somewhat correlated.

The maximum magnitude of the effect for these temperature profiles is an approximately $1 \%$ deviation from the wall temperature so the coefficient in Eqn. 9 is already very small. The absolute values of the temperatures are dominated by the free parameter for the temperatures at the walls, so even a relatively large change in the value of the fitted thermal conductivity can be compensated by a small extra shift in the temperature slip value. For example, fixing the value of the thermal conductivity for system P83 and $F_{x}^{e}=0.0005$ at $\lambda=6$ instead of the fitted value $\lambda=5.6$ results in a channel centre temperature change from $T(0)=1.0024$ to $T(0)=1.00233$ which can easily be compensated with a small shift in the profile height (i.e. from $T_{w}=1.0004$ to $\left.T_{w}=1.00047\right)$ without significantly distorting the curve and while maintaining visual congruity with the plotted data. Therefore, we estimate the true uncertainty to be closer to \pm 0.5 than \pm 0.1 in this case.

The data presented here were very difficult to collect due to the small absolute magnitude of the temperature profile and the extent of statistical sampling required to reduce noise. This justifies the isothermal assumption commonly made for experimental Poiseuille flow, especially for the smallest body force presented here, which is almost indistinguishable from equilibrium.

Fig. 8 shows a comparison between the density oscillations and the residuals for the purely quartic fit to the temperature profile for systems P23 and P11. With the exception of the first peak, where depletion and slip effects are strong, the residuals are strongly anti-correlated with the density oscillations. The residuals for the widest system, P83, showed no trends and, as such, have not been displayed.

Similarly to the case for the velocity profile residuals, the temperature residuals of the P11 system show a much stronger anti-correlation with the density oscillations than the P23 system. This is consistent with the fact that that the steady state temperature profile is the net result of internal energy production due to the work done by the field driving the flow, which is given by $-P_{y x} \dot{\gamma}$ and heat conduction. We expect the nonlinear, nonlocal coupling between the applied external field and the confining field that is responsible for both the shear pressure and the strain rate profiles $[24,25]$ to result in a temperature profile that is strongly dependent on the driving field.

The residuals for the P11 system (right) show a slight trending increase towards the channel-centre which may be caused by strain rate coupling to the heat flux. If this is the case then the strain rate coupling coefficient, $\xi$, is of order 1 , where the (strain rate coupled) heat flux vector and resulting temperature profiles are defined by $[4,6]$,

$$
J_{q}(y)=-\lambda \frac{\partial T(y)}{\partial y}-\xi \frac{\partial \dot{\gamma}^{2}(y)}{\partial y}
$$

and

$$
\begin{aligned}
T(y) & =-\frac{\rho^{2}\left(F_{x}^{e}\right)^{2}}{12 \lambda \eta}\left(y^{4}-\frac{W^{4}}{16}\right) \\
& -\frac{\rho^{2}\left(F_{x}^{e}\right)^{2} \xi}{\lambda \eta^{2}}\left(y^{2}-\frac{W^{2}}{4}\right)+T_{w} .
\end{aligned}
$$

By comparing the coefficients in this equation we find that the contribution of the quadratic term only dominates when $y<\sqrt{12 \xi / \eta}$. For our value of $\xi$ this corresponds to a centre channel region of $-1.73<y<1.73$. Systems P23 and P83 have respectively 3 and 30 times smaller values of the external field. In these cases, the amplitude of the quadratic term in the temperature profile is reduced by factors of 9 and 900 . Therefore, the lack of evidence in these cases might simply be due to the noise in the data eclipsing the magnitude of strain rate coupling effect.

Overall we find that the temperature profile is well described by the continuum prediction given by Eqn. 9; any coupling to the strain rate is weak for this system. In the P83 system, where the effects due to density oscillations are negligible, the fitted equations work extremely well. For systems P23 and P11 the fits could be improved by an appropriate coupling to the density profiles, which should include either the magnitude of the applied external field or the gradient of the strain rate as a factor.

\section{CONCLUSION}

We performed equilibrium simulations of polymer solutions, calculated transport coefficients, and compared them with transport coefficients extracted from nonequilibrium molecular dynamics Poiseuille flow simulations. We found that the Newtonian zero strain rate viscosity represented the velocity profile very well for systems over 20 atomic diameters, with only a slight deviation near the walls due to the polymer depletion effect. However, in channels of 20 atomic diameters and less the perturbation of velocity due to the local density oscillations becomes the dominant factor.

Similarly, we also found that the temperature profiles of systems more than 20 atomic diameters wide were well described by Fourier's law. For narrower channels the density oscillations start to have a major effect on the temperature, however the underlying temperature profile is still well described by the expected quartic equation.

We found some evidence of a small strain rate coupled temperature with the strain rate coupling coefficient $\xi \approx 1$. 
Results from STF NEMD simulations of simple fluids by Baranyai et al. [4] and Ayton et al. [5] et al. indicated a strain rate coupling coefficient of $\xi \approx 1$ which compares well with our result. Ayton et al. also postulated that one must use the so-called 'normal temperature' to calculate the correct value of the strain rate coupling. Our similar results indicate that, at least for the systems and flow rates presented here, the kinetic temperature is an appropriate temperature for calculation of the strain rate coupling.

In contrast to these results, data from NEMD simulations of simple fluids experiencing planar Poiseuille flow by Todd and Evans [6] indicates a strain rate coupling coefficient of $\xi \approx 80$. In their work the temperature was determined instantaneously from the thermal velocities at each timestep using an approximation to the streaming velocity obtained by fitting a polynomial equation to the instantaneous velocity profile. Their large magnitude of $\xi$ may have resulted from an incorrect determination of the streaming velocity due to the exclusion of higher order terms from the fit to the instantaneously sampled velocity profile, which could be very noisy.

The difficulty of investigating the strain rate coupling phenomena in planar Poiseuille flow is that the region in which the coupling term dominates is small. More highly confined channels allow for this region to extend into a relatively larger proportion of the channel, but also introduce density oscillations that significantly perturb the temperature profile. Increasing the chain length of the polymer molecules might lead to a higher strain rate coupling coefficient, allowing the region where the strain rate coupling dominates to extend, such that the effect will be more prominent in wider channels where perturbations due to density oscillations are spatially limited.

Inadequate determination of the streaming velocity can also lead to a spurious contribution to the magnitude of the strain rate coupling in systems where the temperature profile is not correctly determined. For NEMD STF simulations this could be investigated by performing simulations with a larger number of particles per averaging bin, a running average estimate to the streaming velocity, or a post-processed estimate for the streaming velocity. This would ensure a correctly calculated local temperature from which the strain rate coupling coefficient could be determined.

\section{Acknowledgements}

We would like to acknowledge the National Computational Infrastructure (NCI) for use of their supercomputing facilities. Adrian Menzel would like to thank the Australian Government for an Australian Postgraduate Award, and RMIT University which hosts his post-graduate studies.

This paper is dedicated to W.G. Hoover on the occasion of his $80^{\text {th }}$ birthday.

\section{References}

[1] M.D. Graham, Fluid Dynamics of Dissolved Polymer Molecules in Confined Geometries, Annu. Rev. Fluid Mech. 43(1), 273-298 (2011).

[2] T. Kairn, Microscopic Simulation of Colloidal Flow in Thin Channels, PhD Thesis, Royal Melbourne Institute of Technology 2005.

[3] I.K. Snook, P.J. Daivis, T. Kairn, The flow of colloids and polymers in channels simulated using non-equilibrium molecular dynamics, J. Physics - Condensed Matter 20(40), 404211 (2008).

[4] A. Baranyai, D.J. Evans, P.J. Daivis, Isothermal shear-induced heat flow, Phys. Rev. A 46(12), 7593-7600 (1992).

[5] G. Atyon, O.G. Jepps, D.J. Evans, On the validity of Fourier's law in systems with spatially varying strain rates, Mol. Phys. 96(6), 915-920 (1999).

[6] B.D. Todd, D.J. Evans, Temperature profile for Poiseuille flow, Phys. Rev. E 55(3), 2800-2807 (1997).

[7] S. Hess, M.M. Mansour, Temperature profile of a dilute gas undergoing a plane Poiseuille flow, Physica A 272(3), 481496 (1999).

[8] K.P. Travis, K.E. Gubbins, Poiseuille flow of Lennard-Jones fluids in narrow slit pores, J. Chem. Phys. 112(4), 1984-1994 (2000).

[9] H. Ma, M.D. Graham, Theory of shear-induced migration in dilute polymer solutions near solid boundaries, Phys. Fluids 17(8), 1-13 (2005).

[10] M.L. Matin, P.J. Daivis, B.D. Todd, Comparison of planar shear flow and planar elongational flow for systems of small molecules, J. Chem. Phys. 113(20), 9122-9131 (2000).

[11] T. Kairn, P.J. Daivis, M.L. Matin, I.K. Snook, Concentration dependence of viscometric properties of model short chain polymer solutions, Polymer 45(7), 2453-2464 (2004).

[12] T. Kairn, P.J. Daivis, M.L. Matin, I.K. Snook, Effects of concentration on steady-state viscometric properties of short chain polymer solutions over the entire concentration range, Int. J. Thermophys. 25(4), 1075-1084 (2004).

[13] J.D. Weeks, D. Chandler, H.C. Andersen, Role of Repulsive Forces in Determining the Equilibrium Structure of Simple Liquids, J. Chem. Phys. 54(12), 5237-5247 (1971).

[14] J.E. Jones, On the Determination of Molecular Fields. II. From the Equation of State of a Gas, Proc. R. Soc. A Math. Phys. Eng. Sci. 106(738), 463-477 (1924).

[15] B.D. Todd, P.J. Daivis, Nonequilibrium molecular dynamics, Cambridge University Press 2017.

[16] C.W. Gear, The Numerical Integration of Ordinary Differential Equations, Math. Comput. 21(98), 146-156 (1967).

[17] M.S. Green, Markoff Random Processes and the Statistical Mechanics of Time-Dependent Phenomena. II. Irreversible Processes in Fluids, J. Chem. Phys. 22(3), 398 (1954).

[18] R. Kubo, Statistical-mechanical theory of irreversible processes. I. general theory and simple applications to magnetic and conduction problems, J. Phys. Soc. Japan 12(6), 570-586 (1957).

[19] P.J. Daivis, Thermodynamic relationships for shearing linear viscoelastic fluids, J. Nonnewton. Fluid Mech. 152(1-3), 120-128 (2008).

[20] S.R. De Groot, P. Mazur, Non-equilibrium thermodynamics, Courier Dover Publications 2013.

[21] D.J. Evans, G.P. Morriss, Statistical Mechanics of Nonequilbrium Liquids, CUP 2008.

[22] P.J. Daivis, B.D. Todd, A simple, direct derivation and proof of the validity of the SLLOD equations of motion for generalized homogeneous flows, J. Chem. Phys. 124, 194103 (2006). 
[23] A.B. Metzner, Y. Cohen, C. Rangel-Nafaile, Inhomogeneous flows of non-newtonian fluids: generation of spatial concentration gradients, J. Nonnewton. Fluid Mech. 5(C), 449-462 (1979).

[24] K.S. Glavatskiy, B.A. Dalton, P.J. Daivis, B.D. Todd, Nonlocal response functions for predicting shear flow of strongly inhomogeneous fluids. I. Sinusoidally driven shear and sinusoidally driven inhomogeneity, Phys. Rev. E 91062132 (2015).

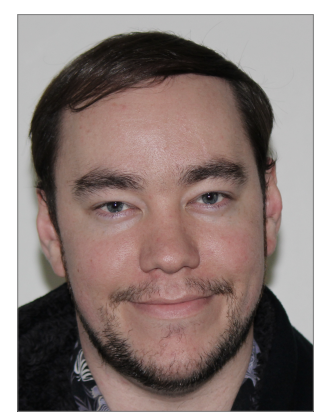

Adrian Menzel received his Bachelor of Science (Physics) from RMIT in 2011 and subsequently went on to complete an honours year in 2013. He is currently undertaking his doctorate studies under the supervision of Prof. Daivis and Prof. Todd. His research interests are in non-equilibrium molecular dynamics, statistical mechanics, thermodynamics, and rheology, as well as scientific visualisation.

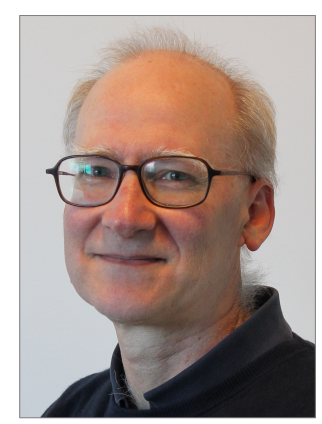

Peter J. Daivis has been a professor of physics at RMIT University, Australia, since 2011. He completed undergraduate and master's degrees in applied physics at RMIT University, and a graduate diploma in applied colloid science at Swinburne Institute of Technology, Australia. After completing his PhD degree in physics at Massey University, New Zealand, he was appointed to a postdoctoral position at The Australian National University. His research interests include experimental, computational, and theoretical studies of soft matter, using applications of nonequilibrium statistical and thermal physics, computational nanofluidics, and generalised hydrodynamics.

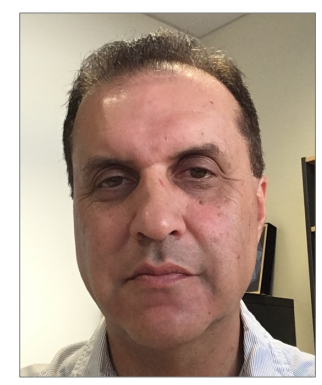

Billy Todd is a professor and chair of the Department of Mathematics at Swinburne University of Technology, Australia. He completed his undergraduate and postgraduate studies in physics at The University of Western Australia and Murdoch University, Australia. He then completed postdoctoral appointments at the University of Cambridge, UK, and The Australian National University, before moving to the Commonwealth Scientific and Industrial Research Organisation (CSIRO) in Melbourne, Australia, in 1996. His research interests include statistical mechanics, nonequilibrium molecular dynamics, and computational nanofluidics. He is a Fellow of the Australian Institute of Physics and a former President of the Australian Society of Rheology. 